\title{
Acting while perceiving: assimilation precedes contrast
}

\author{
Marc Grosjean · Jan Zwickel $\cdot$ Wolfgang Prinz
}

Received: 1 October 2007 / Accepted: 1 March 2008 / Published online: 26 March 2008

(c) Springer-Verlag 2008

\begin{abstract}
To explore the nature of specific interactions between concurrent perception and action, participants were asked to move one of their hands in a certain direction while simultaneously observing an independent stimulus motion of a (dis)similar direction. The kinematics of the hand trajectories revealed a form of contrast effect (CE) in that the produced directions were biased away from the perceived directions ("Experiment 1"). Specifically, the endpoints of horizontal movements were lower when having watched an upward as opposed to a downward motion. However, when participants moved under higher speed constraints and were not presented with the stimulus motion prior to initiating their movements, the $\mathrm{CE}$ was preceded by an assimilation effect, i.e., movements were biased toward the stimulus motion directions ("Experiment 2"). These findings extend those of related studies by showing that CEs of this type actually correspond to the second phase of a bi-phasic pattern of specific perception-action interference.
\end{abstract}

\section{Grosjean · J. Zwickel · W. Prinz}

Department of Psychology,

Max Planck Institute for Human Cognitive and Brain Sciences, Munich, Germany

\section{Grosjean $(\bowtie)$}

Leibniz Research Centre for Working Environment and Human Factors, Ardeystrasse 67, 44139 Dortmund, Germany

e-mail: grosjean@ifado.de

Present Address:

J. Zwickel

Neuro-Cognitive Psychology Unit, Department of Psychology, Ludwig Maximilian University Munich, Leopoldstrasse 13, 80802 Munich, Germany

\section{Introduction}

Many daily activities involve performing an action while simultaneously encoding one or more perceptual events. In contrast to most experimental tasks aimed at studying specific interactions between perception and action (for overviews, see Prinz \& Hommel, 2002; Proctor \& Reeve, 1990), such situations are characterized by the fact that what is perceived temporally overlaps with ongoing action for relatively long periods of time and does not (necessarily) specify what movements are to be concurrently produced. As common as these situations may seem, they have only recently gained interest.

An illustrative example comes from a study by Jacobs and Shiffrar (2005), in which they asked people to judge the speed of point-light walkers while either standing, bicycling, or walking themselves. They found that speed judgments were less accurate during walking than during bicycling or standing. This finding not only demonstrates that action can interfere with perception, but that the nature of interference depends on whether what is produced (e.g., walking) shares representational features with what is perceived (e.g., seeing someone else walk; for related effects, see Hamilton, Wolpert, \& Frith, 2004; Schubö, Prinz, \& Aschersleben, 2004; Wohlschläger, 2000; Zwickel, Grosjean, \& Prinz, 2007). Interference effects of this type are referred to as specific, as opposed to unspecific, because they relate to changes in performance that are determined by the relationship or degree of feature overlap (at a representational level) between the contents of perception and action (Müsseler, 1999).

The purpose of the present study was to further this line of investigation by exploring how perception specifically interacts with action under such "concurrent" conditions. To do so, we relied on a task introduced by Schubö, Aschersleben, and Prinz (2001) in which they showed that the simul- 
taneous perception and production of movements leads to a repulsion between perception and action: movements were biased away from the stimulus motions that were simultaneously perceived. In the current study, we sought to explore the time course of this effect and, in particular, establish whether it is actually preceded by an attraction between what is perceived and produced. That is, a movement bias toward the concurrently observed motions.

Unlike conventional stimulus-response (S-R) compatibility-type tasks (e.g., Proctor \& Reeve, 1990), Schubö et al.'s (2001) paradigm was developed to investigate specific interactions that arise when what is perceived is functionally unrelated to what is simultaneously produced. These terms are used to refer to any situation in which the movement required by the participant is not specified nor consists of a reaction to the stimulus that is concurrently presented (see also Zwickel et al., 2007). Their paradigm also involved more dynamic stimuli and movements than those usually encountered in the $\mathrm{S}-\mathrm{R}$ compatibility literature (for similar efforts see, e.g., Brass, Bekkering, Wohlschläger, \& Prinz, 2000; Chua \& Weeks, 1997; Grosjean \& Mordkoff, 2001). On a given trial $n$, the currently presented stimulus motion $\left(S_{n}\right)$ specified the required movement $\left(M_{n+1}\right)$ for the subsequent trial, whereas the currently required movement $\left(M_{n}\right)$ was specified by the stimulus $\left(S_{n-1}\right)$ presented on the previous trial. The stimulus consisted of a dot that moved along a sinusoidal trajectory of either a small, medium, or large amplitude, and the required movement consisted of drawing on a graphics tablet sinusoidal trajectories of one of the same (i.e., small, medium, or large) amplitudes without visual feedback. Thus, participants were required to perform a previously specified action while simultaneously encoding a functionally unrelated, but feature-overlapping, stimulus motion.

The question of interest in this task was whether the perception of the amplitude of $S_{n}$ would specifically interact with the produced $M_{n}$ amplitude. This was the case. When participants were required to produce a medium-amplitude trajectory, watching a small-amplitude trajectory led to an increase in movement amplitude, whereas watching a large-amplitude trajectory led to a decrease in movement amplitude. Although Schubö et al. (2001) did not perform any fine-grained analyses of the time course of their effect, they did report that it was already present at the first extreme value of the sinusoidal movement trajectories, which corresponded to $\sim 600-700 \mathrm{~ms}$ after movement onset (as inferred from their Fig. 2).

These results were taken as evidence of a new type of specific perception-action interaction that can be characterized as a contrast effect (CE). The effect is contrast-like in that it is typified by a repulsion (as opposed to an attraction) between perception and action. Schubö et al. (2001) accounted for their effect by drawing inspiration from models of reaching in the presence of stationary distractor objects (e.g., Tipper, Howard, \& Houghton, 1998). In particular, they proposed that people try to minimize the amount of interference between perception and action by engaging in the mutual partial inhibition of the representations or features that overlap for what is perceived and produced (i.e., the codes responsible for representing the amplitudes of the motions and movements, respectively). This, in turn, causes the representations underlying the perceived motion and produced movement to "shift away" from each other, thereby leading to a CE.

Schubö et al.'s (2001) study demonstrates how the simultaneous perception and production of functionally unrelated events leads to interference (contrast) effects in movement execution. We sought to explore the time course of the CE by determining at what point after movement onset it arises. There are two main reasons why this is of interest. First, and more generally, establishing the time course of such interactions has become critical for developing and testing relevant models of the perception-action interface (e.g., De Jong, Liang, \& Lauber, 1994; Welsh \& Elliott, 2004; Wiegand \& Wascher, 2005). Second, when perceptual and motor events share features at a representational level, as is typically the case under compatible S-R conditions, assimilation (facilitation) effects rather than contrast (interference) effects have generally been observed in both discrete (for an overview, see Hommel \& Prinz, 1997) and continuous perception-action tasks (e.g., Chua \& Weeks, 1997; Keller \& Burnham, 2005; Michaels \& Stins, 1997). In light of these findings, the CEs obtained by Schubö et al. (2001) were to some extent unexpected.

Of particular relevance to this discussion is a study by Whitney, Westwood, and Goodale (2003), in which they asked people to reach as quickly as possible to a briefly flashed stationary target in the presence of an unrelated moving grating. They obtained a form of assimilation effect (AE): hand trajectories initially deviated in the direction of the motion as early as $\sim 120 \mathrm{~ms}$ after motion onset. Although their task was in many ways different from that of the Schubö et al. (2001), their findings further suggest that perceived motions can have an assimilatory influence on ongoing movements. Thus, by exploring the time course of the CEs, we sought to establish whether they were actually preceded by something akin to an AE.

\section{Experiment 1}

In addition to exploring the time course of the $\mathrm{CE}$, the aim of the first experiment was also to generalize the findings of Schubö et al. (2001) to the overlapping motion/movement dimension of direction and to the perception of non-biological stimulus motions. To do so, we asked participants to move one of their hands along a straight line in a certain 
direction while simultaneously observing an independent stimulus motion of a (dis)similar direction. However, in contrast to Schubö et al.'s study, in which the stimulus motions had biological kinematics, we used constant (nonbiological) stimulus velocity profiles. For the type of (linear) trajectories employed here, biological velocity profiles would have been roughly bell-shaped (e.g., Beggs \& Howarth, 1972; Morasso, 1981).

The purpose of relying on stimulus motions with nonbiological kinematics was twofold. First, it has the advantage of discouraging participants from interpreting the motion of the stimulus as a consequence of the movement that they are concurrently executing, which is essential for investigating interference effects that arise during the concurrent perception and production of functionally unrelated events. Second, it is well established that biological motions are processed differently than non-biological motions (e.g., Viviani, 2002). Moreover, interference effects obtained in paradigms similar to Schubö et al.'s (2001) have been linked to the involvement of the motor system in the visual processing of biological motion (e.g., Kilner, Paulignan, \& Blakemore, 2003).

Thus, if the CE observed by Schubö et al. (2001) is constrained to the processing of biological motion, then there should be no effect of the observed motion direction on the produced movement direction. Alternatively, based on the findings of Whitney et al. (2003) and others (e.g., Chua \& Weeks, 1997; Keller \& Burnham, 2005; Michaels \& Stins, 1997), the present paradigm may reveal an AE of perception on action, rather than or in addition to a CE.

Method

\section{Participants}

Twenty individuals (mean age $=26.15$ years; age range $=21-37$ years) participated in the experiment. In this, as well as in the subsequent experiment, all participants were paid $18 €$ for their time, were right handed, had normal or corrected-to-normal vision, and were not informed of the purpose of the study.

\section{Apparatus, stimuli, and movements}

The experiment took place in a sound-attenuated and dimly-illuminated chamber. The presentation of the stimuli (Ss) and recording of movements (Ms) were controlled by an IBM-compatible microcomputer connected to a $21 \mathrm{in}$. color monitor with the refresh rate set at $70 \mathrm{~Hz}$. As illustrated in Fig. 1a, the stimuli consisted of a circle moving along an imaginary straight line from the center of an imaginary circle to its perimeter in one of three possible directions: upward, horizontal, or downward. The circle $(6 \mathrm{~mm}$
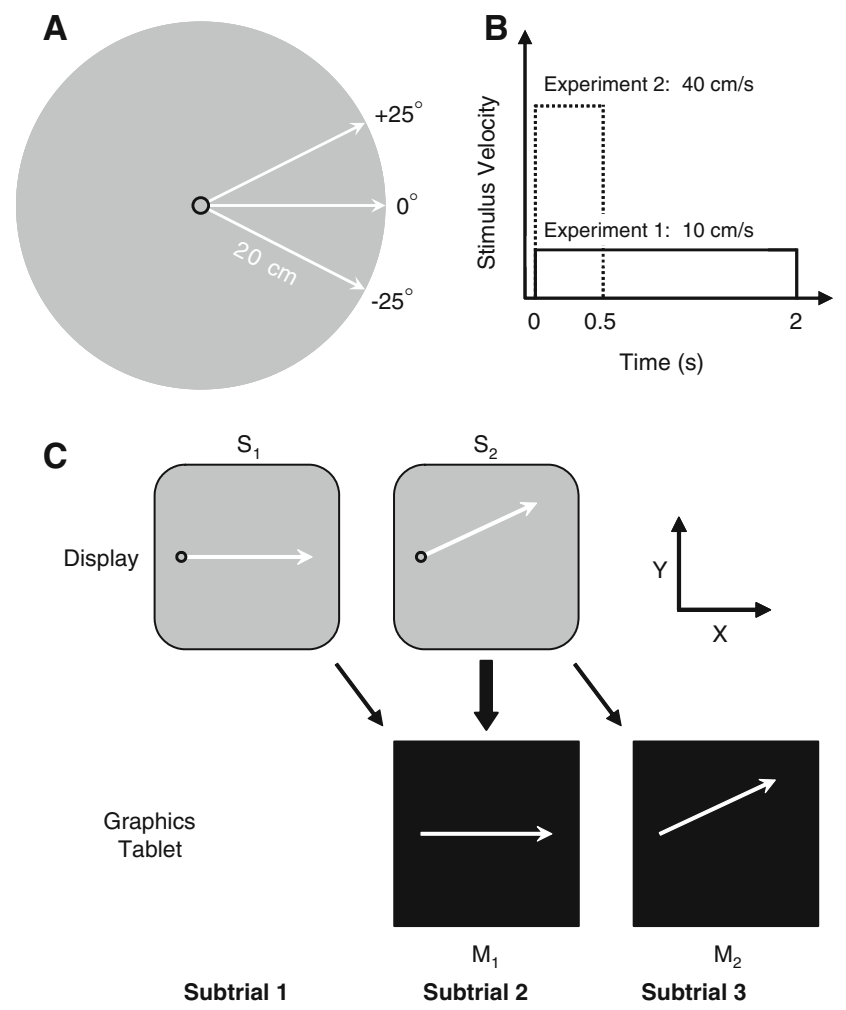

Fig. 1 Schematic of the (a) stimulus and movement trajectories, (b) stimulus velocity profiles, and (c) structure of a trial and the coordinate system that was used (see text for details). $S$ stimulus, $M$ movement

in diameter) was presented in red on a black background and did not leave a trace on the display as it moved. The trajectory length (i.e., radius of the imaginary circle) was $20 \mathrm{~cm}$ and the angles by which the upward and downward trajectories deviated from the horizontal one were $+25^{\circ}$ and $-25^{\circ}$, respectively. The circle always departed from the same start position that was vertically centered in the display and shifted to the left of its vertical midline by half of the trajectory length (i.e., $10 \mathrm{~cm}$; see Fig. 1a, c). Given the approximate viewing distance of $60 \mathrm{~cm}$, the circle subtended $0.57^{\circ}$ and traversed a path of $18.92^{\circ}$ of visual angle. In line with Schubö et al. (2001), the duration of the stimulus motion was held constant at $2 \mathrm{~s}$. However, in contrast to their experiments, stimulus velocity was non-biological in that it was set to the constant value of $10 \mathrm{~cm} / \mathrm{s}$ (i.e., $9.46 \%$; see Fig. 1b).

As for the movements, participants were asked to draw with their right hand the same upward, horizontal, and downward trajectories as those employed for the stimuli. Movements were performed by displacing a stylus on a Wacom Ultrapad A3E graphics tablet. The $x$ and $y$ positions of the hand-held stylus were sampled in synchrony with the monitor's refresh rate (i.e., $70 \mathrm{~Hz}$ ). The graphics tablet was centered with respect to and horizontally placed directly below the monitor, and was covered with a board 
such that no direct visual feedback of the stylus or hand was provided. The start position for the hand movements on the tablet was horizontally aligned with the constant stimulus start position. Given the placement of the tablet, the stylus was always moved within the horizontal plane. Thus, "upward" and "downward" movement trajectories actually corresponded to hand movements that went away from and towards to the participants' body, respectively. This mapping was learned during a practice phase in which off-line feedback of the produced movements was provided on the monitor (see the next section for more details).

\section{Design and procedure}

Participants were tested in a single session that lasted about $2 \mathrm{~h}$. The experiment began with oral instructions in which participants were informed that they would be seeing motions of a stimulus that they would have to subsequently reproduce as accurately as possible on the graphics tablet. Each trial was divided into three subtrials (see Fig. 1c). The stimulus on a given subtrial specified the movement to be performed on the subsequent subtrial (as indicated by the oblique arrows in the figure). However, on the first subtrial, $S_{1}$ was presented without the concurrent production of a movement to ensure that it was perceived without being influenced by ongoing motor activity. Then, on the second subtrial, $S_{2}$ was presented during the production of $M_{1}$. In this way, the execution of $M_{1}$ should reflect the influence of the perception of $S_{2}$ (as indicated by the block arrow in Fig. 1c). Finally, on the third subtrial, $M_{2}$ was produced in isolation, thereby avoiding interference from ongoing perceptual activity that would have been induced by the presentation of a new stimulus. The only purpose of including $M_{2}$ was to make certain that participants had adequately perceived $S_{2}$, which could have otherwise been ignored.

Each subtrial began with the presentation of the stimulus (i.e., the red circle) at the start position. On the first subtrial, the stimulus was also filled in red, indicating to participants that all that was required of them was to watch the stimulus motion. One second after the stimulus appeared, a first tone $(1,760 \mathrm{~Hz}, 20 \mathrm{~ms})$ was played and the stimulus started to move along one of the three possible trajectories. After $2 \mathrm{~s}$, the stimulus motion ended and a second tone was played $(880 \mathrm{~Hz}, 20 \mathrm{~ms})$. The stimulus then remained at the final trajectory position for another $1 \mathrm{~s}$, after which the display went blank and a third tone ( $440 \mathrm{~Hz}, 20 \mathrm{~ms}$ ) was played to indicate the end of the subtrial. After a $1 \mathrm{~s}$ inter-subtrial interval, the stimulus for the second subtrial was presented along with a cursor [a white disk with a diameter of $2 \mathrm{~mm}\left(0.19^{\circ}\right.$ of visual angle $\left.)\right]$ representing online the position of the stylus on the graphics tablet. The gain was adjusted such that there was a 1:1 mapping between movement of the stylus on the graphics tablet and the corresponding movement of the cursor. To initiate the second subtrial, participants had to move the cursor into the start position (i.e., into the stimulus). Once the cursor had remained there for $1 \mathrm{~s}$, the cursor disappeared, the first tone was played, the stimulus was set into motion, and the coordinates of the stylus started to be saved. Participants were instructed to begin their movement with the onset of the first tone and to terminate it with the onset of the second tone. That is, the target movement duration was $2 \mathrm{~s}$. As before, the end of the subtrial was indicated by the third tone being played $1 \mathrm{~s}$ after stimulus motion offset. This also marked the end of the graphics tablet recording, which lasted for a total of $3 \mathrm{~s}$. After the inter-subtrial interval, the stimulus and the cursor were presented for the third subtrial. This subtrial was identical to the second subtrial, except that the stimulus stayed at the start position after the first tone was played and the cursor had disappeared (i.e., there was no stimulus motion while the participants moved). After an inter-trial interval of $1 \mathrm{~s}$, the stimulus for the first subtrial of the next trial was presented. At the end of a block, a minimum break of $10 \mathrm{~s}$ was imposed before participants could self-initiate the following block whenever they felt ready.

To familiarize the participants with the task and particularly the to-be-produced movements, the experiment began with two practice blocks in which $S_{1}$ and $S_{2}$ were identical for each trial. Therefore, during the second subtrial, participants always had to produce the same trajectory as they concurrently saw. During these practice blocks, participants received end-of-subtrial feedback for $2 \mathrm{~s}$ consisting of a depiction of the required trajectory as a red line and the trajectory they had produced presented in white. It was via this off-line feedback that participants learned the mapping between their movements and those presented on the display. Each of the practice blocks was 15 trials long, corresponding to three $S$ directions (upward, horizontal, downward) $\times 5$ repetitions presented in a pseudo-random sequence.

The participants then performed 14 experimental blocks. For these blocks, $S_{1}$ and $S_{2}$ were only identical on onethird of the trials. Therefore, during the second subtrial, participants had to produce a different trajectory from what they saw on twothird of the trials. Each of the experimental blocks was 18 trials long, corresponding to three $S_{1}$ directions (upward, horizontal, downward) $\times 3 S_{2}$ directions (upward, horizontal, downward) $\times 2$ repetitions presented in a pseudo-random order. To assist the participants in adjusting to this more demanding version of the task, the first experimental block included the same end-of-subtrial trajectory feedback as that provided during the practice blocks. From that point onwards, however, no feedback was ever provided. 


\section{Data analysis}

Data from the practice blocks and the first experimental block were discarded. To avoid any problems associated with range effects (see Schubö et al., 2001 for a discussion of this issue), the analysis was further restricted to trials in which horizontal movements were performed on the second subtrial (i.e., when $S_{1}$ was horizontal). Given the present experimental design (see above), this resulted in 26 remaining trials for each condition and participant. Moreover, to ensure that participants had adequately produced the trajectories required of them, movements were excluded when participants lifted the stylus, reversed movement direction, and when the length and angle of the trajectory at movement offset were not within $\pm 50 \%$ of the required trajectory length and angle. Finally, $M_{1}$ was also discarded when $M_{2}$ met any of these exclusion criteria because it was the only way to be certain that participants had adequately perceived $S_{2}$. This was the sole purpose of analyzing the second movements and are therefore not considered any further.

The movement trajectories were each analyzed by first realigning the $x$ and $y$ stylus values to a common $(0,0)$ coordinate position. Tangential velocity profiles were then obtained through numerical derivation. Movement onset and offset were defined as the first moments in time at which tangential velocity reached $5 \%$ of peak tangential velocity before and after this peak, respectively. On the basis of these temporal markers, movement initiation time (=time of movement onset - time of stimulus onset), movement time (=time of movement offset - time of movement onset), and the $y$ (i.e., vertical) position at movement offset were determined for each condition and participant. This latter variable was of particular interest, as it should reveal the overall magnitude of the interference effect. These variables were each submitted to a separate one-way repeated-measures analysis of variance (ANOVA) with stimulus direction (upward, horizontal, downward) as a within-subjects factor.

For the purpose of movement trajectory averaging and exploring the time course of the interference effect, the trajectory for each trial was normalized to 101 time-slices $(0$ $100 \%)$. This was done by resampling the time vector of each trajectory between movement onset and offset at 101 equally time-spaced values and computing, using piecewise cubic spline interpolation (as implemented in the "interp1" function in MATLAB), the corresponding stylus coordinate values (separately for the $x$ and $y$ coordinate vectors) $)^{1}$. To

\footnotetext{
${ }^{1}$ Cubic spline interpolation was used because of the curvature present in the hand trajectories and the fact that it incurs smaller errors (on average) than other forms of interpolation (e.g. linear). However, to be certain that the results did not depend on this particular form of interpolation, the same analyses were also performed using linear interpolation. When conducted in this way, the analyses did not yield any differences in the overall pattern of results.
}

Table 1 Mean movement initiation time (in ms), movement time (in $\mathrm{ms}$ ), and $Y$ position at movement offset (in $\mathrm{mm}$ ) for horizontal movements from the second subtrials as a function of the concurrently perceived stimulus direction (upward, horizontal, downward) for "Experiments 1 and 2"

\begin{tabular}{llll}
\hline Experiment and measure & \multicolumn{3}{l}{ Stimulus direction } \\
\cline { 2 - 4 } & Upward & Horizontal & Downward \\
\hline "Experiment 1" & & & \\
Movement initiation time & 226 & 228 & 233 \\
Movement time & 1996 & 2004 & 1989 \\
$Y$ position at movement offset & -2.08 & 2.96 & 5.56 \\
"Experiment 2" & & & \\
Movement time & 769 & 716 & 751 \\
$Y$ Position at movement offset & -15.69 & -5.27 & 0.71 \\
\hline
\end{tabular}

Mean movement initiation time is not provided for "Experiment 2" because movement recording began with movement onset

investigate the time course of the interference effect, the difference in $y$ position $(\Delta Y)$ at each normalized time sample between mean horizontal movements performed under upward and downward stimulus conditions was calculated for each participant. Positive and negative $\Delta Y$ values correspond to movement trajectories that deviated in the direction of stimulus motion (assimilation) or deviated away from the stimulus motion (contrast), respectively. A twotailed paired $t$ test was then performed at each normalized time sample in order to determine at which point the mean trajectories began to differ from each other (for the use of analogous methods, see Brenner \& Smeets, 1997; Spivey, Grosjean, \& Knoblich, 2005).

For all analyses, $\alpha$ was set to 0.05 and, when necessary, violations of sphericity were corrected for using the Greenhouse-Geisser $\varepsilon$ (to facilitate reading, the uncorrected degrees of freedom are provided).

\section{Results and discussion}

The exclusion criteria led to a total of $5.83 \%$ of discarded trials. Table 1 presents mean movement initiation time and movement time as function of the concurrently perceived stimulus direction. Movement initiation times were relatively short (overall mean $=229 \mathrm{~ms}$ ) and movement times (overall mean $=1996 \mathrm{~ms}$ ) nicely approximated the target duration of $2 \mathrm{~s}$. However, neither of these measures significantly varied as a function of the concurrently perceived stimulus direction (both $P$ 's $>0.09$ ).

Figure 2a depicts the mean horizontal movement trajectories for the three stimulus directions. As can be seen in the figure, the trajectories showed a certain degree of curvature that bent away from the participants' body, which is a common finding for such lateral hand movements (for a 

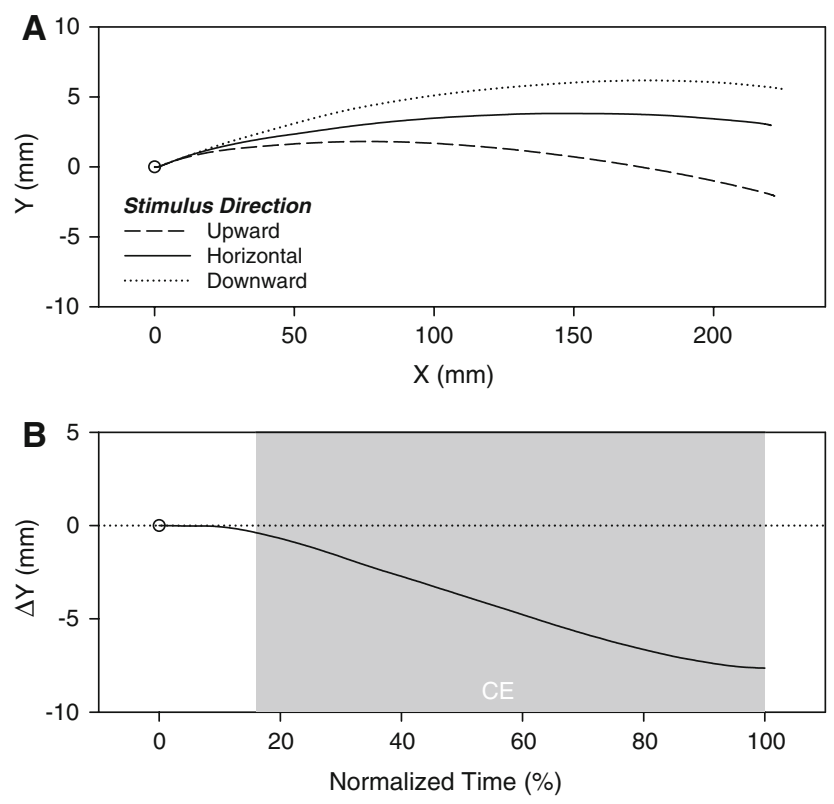

Fig. 2 Data from the second subtrial movements in "Experiment 1". a Mean horizontal movement trajectories as a function of the concurrently perceived stimulus direction (upward, horizontal, downward). b Mean difference in $y$ position $(\Delta Y)$ between horizontal movement trajectories produced under upward and downward stimulus direction conditions as a function of normalized time. Positive and negative $\Delta Y$ values correspond to assimilation and contrast, respectively. The shaded region indicates the normalized time values at which $\Delta Y \mathrm{~s}$ are significantly different from 0 , that is the values at which an assimilation $(A E)$ or contrast effect $(C E)$ was present

recent example, see Saunders \& Knill, 2003). More interestingly, the trajectories for the different stimulus directions fanned apart from each other over time. In particular, the trajectories for the upward and downward conditions deviated away from the perceived motion directions. By the end of the movement, having watched an upward motion led to a $7.64 \mathrm{~mm}$ lower movement endpoint than having watched a downward motion, whereas the horizontal motion condition led to an intermediate final position (see Table 1). This effect of stimulus direction on the mean $y$ position at movement offset was significant, $F(2,38)=27.26, P<0.001$, $\eta_{\mathrm{p}}^{2}=0.59, \varepsilon=0.88$.

These findings replicate and extend those of Schubö et al. (2001) for the overlapping motion/movement dimension of direction. Moreover, this CE was obtained with constant stimulus motion velocity profiles, which demonstrates that interference effects of this type are not contingent upon the processing of biological motion, in contrast to what has been previously suggested in the context of similar tasks (e.g., Kilner et al., 2003).

The time course of the CE is captured in Fig. 2b, which presents the mean difference in $y$ position between the trajectories produced under upward and downward stimulus conditions as a function of normalized time. As indicated by the shaded region in the figure, the trajectories began to significantly diverge from each other at $17 \%$ of normalized time. This value corresponds to a mean absolute time of $339 \mathrm{~ms}$ after movement onset and $569 \mathrm{~ms}$ after stimulus motion onset (as computed on the basis of the mean movement initiation and movement times for the upward and downward stimulus directions). As one would expect, the $\mathrm{CE}$ takes time to reveal itself in the movement trajectories.

These results demonstrate that CEs of this type generalize to the overlapping dimension of direction and are not confined to the processing of biological motions. However, in contrast to what might have been expected on the basis of related studies (e.g., Whitney et al., 2003), no evidence of an $\mathrm{AE}$ of motion perception on movement production was observed.

\section{Experiment 2}

The goal of "Experiment 2" was to alter the current paradigm in such a way that an $\mathrm{AE}$, if at all present, would have better chances of being revealed. One reason why no AE was observed in "Experiment 1"or in previous studies (Schubö et al., 2001) is that participants may have attempted to counteract this (anticipated) attraction effect. Indeed, given that participants were instructed to reproduce the previously seen motions as accurately as possible, they may have tried to adjust the direction of their trajectories in order to avoid moving in the direction of the stimulus motion. In addition, the movements were performed relatively slowly and the stimulus motion started on average $\sim 230 \mathrm{~ms}$ prior to movement onset. Thus, participants would have had enough time to perform such adjustments (e.g., Desmurget \& Grafton, 2000; Paillard, 1996). This possibility actually suggests that the CEs may be strategic in origin, an issue we return to in "General discussion".

To maximize the chances of revealing a (potential) AE, the paradigm used in "Experiment 1" was first modified by shortening the stimulus motion duration and target movement duration from 2,000 to $500 \mathrm{~ms}$. By increasing movement speed, larger distances will be covered by the participants prior to the time at which they could try to counteract any anticipated or perceived interference effects. This should provide a larger spatial window for detecting an AE. The second modification involved having the stimulus motion start with movement onset. In this way, participants could never see in which direction the stimulus was moving prior to initiating their own movement, which should eliminate the possibility of engaging in counteractive adjustments early in the movement trajectories. 


\section{Method}

A new group of 20 individuals (mean age $=24.50$ years; age range $=17-34$ years) participated in the experiment. The method was identical to that of "Experiment 1", except for two changes. First, the stimulus motion duration and the target movement duration were reduced to $500 \mathrm{~ms}$ (see Fig. 1b). Because the length of the stimulus trajectories was the same as in "Experiment 1", stimulus velocity was accordingly increased to $40 \mathrm{~cm} / \mathrm{s}$ (i.e., $37.84 \%$ ). Second, participants self-initiated the onset of movement recording and stimulus motion (for the second subtrial). As before, after having held the cursor in the start position for $1 \mathrm{~s}$, the first tone sounded. This indicated to the participants that they could initiate their movement whenever they felt ready. As soon as they left the start position, the cursor disappeared, the stimulus was set into motion (on the second subtrial only), and the tablet recording started and lasted for $1 \mathrm{~s}$. Because movement recording began with movement onset, there are no movement initiation times to report.

\section{Results and discussion}

A total of $10.30 \%$ of trials were discarded using the exclusion criteria. The increase in the number of excluded trials with respect to "Experiment 1" can most likely be attributed to the added movement variability that was brought about by shortening the target movement duration (e.g., see Schmidt \& Lee, 1999).

Mean movement times (overall mean $=745 \mathrm{~ms}$ ) overshot the target duration of $500 \mathrm{~ms}$ (see Table 1). More noteworthy was that movement times were longer under upward and downward stimulus direction conditions than under the horizontal condition. This effect of stimulus direction was significant, $F(2,38)=17.51, P<0.001$, $\eta_{\mathrm{p}}{ }^{2}=0.48, \varepsilon=0.73$, and is reminiscent of the response slowing that is typically observed under spatially incompatible relationships between an irrelevant stimulus location/ direction and a relevant response location/direction (e.g., Simon, 1990). Recall, however, that an effect of stimulus direction on movement time was absent in "Experiment 1". This could be due to the absence of strong movement speed constraints and/or the fact that the stimulus motion started prior to movement onset in that experiment (for a detailed discussion of when such compatibility effects arise in movement times, see Rubichi, Nicoletti, Umiltà, \& Zorzi, 2000).

Mean horizontal trajectories are presented in Fig. 3a. As in the previous experiment, the trajectories started to deviate away from the stimulus directions at a certain point in time, such that having watched a downward motion led to a $16.40 \mathrm{~mm}$ higher movement endpoint than having watched an upward motion. The horizontal motion condition again
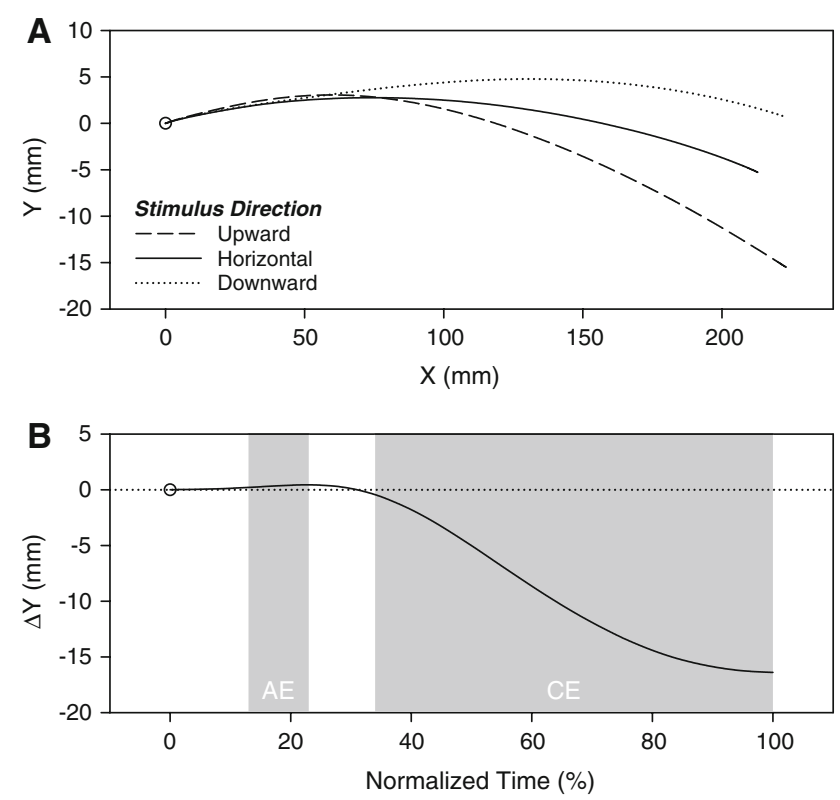

Fig. 3 Data from the second subtrial movements in "Experiment 2". a Mean horizontal movement trajectories as a function of the concurrently perceived stimulus direction (upward, horizontal, downward). b Mean difference in $y$ position $(\Delta Y)$ between horizontal movement trajectories produced under upward and downward stimulus direction conditions as a function of normalized time. Positive and negative $\Delta Y$ values correspond to assimilation and contrast, respectively. The shaded regions indicate the normalized time values at which $\Delta Y \mathrm{~s}$ are significantly different from 0 , that is the values at which an assimilation $(A E)$ or contrast effect $(C E)$ was present

led to an intermediate final position (see Table 1). This pattern was born out in the ANOVA, which yielded a significant effect of stimulus direction on the mean $y$ position at movement offset, $F(2,38)=93.84, P<0.001, \eta_{\mathrm{p}}^{2}=0.83$, $\varepsilon=0.63$. Figure $3 \mathrm{~b}$ presents the time course of the effect. Recall that positive $\Delta Y$ values correspond to movement trajectories that deviated in the direction of stimulus motion (AE) and negative values correspond to trajectories that deviated away from the stimulus motion (CE). As can be seen in the figure, the trajectories began significantly deviating away from the stimulus motions at $35 \%$ of normalized time, which refers to a mean absolute time of $266 \mathrm{~ms}$ after movement/motion onset (note that the absolute times reported here slightly underestimate the actual times because movement recording started at movement onset, i.e., at the moment the stylus left the start position).

What can also been seen by closely inspecting the left portions of Fig. 3a and $\mathrm{b}$ is that prior to deviating away from the stimulus motion directions, the movement trajectories initially deviated towards the motion directions. As indicated by the left shaded region in Fig. 3b, an AE was actually present from 14 to $24 \%$ of normalized time. These values correspond to mean absolute times of 106 and $182 \mathrm{~ms}$ after movement/motion onset, respectively. Given 
that the moment at which the AE reached its peak may have varied between individuals, we further analyzed this effect by searching for the maximum $\mathrm{AE}$ for each participant. This involved finding for each participant the maximum positive deviation in the $\Delta Y$ values that was preceded by six increasing values and followed by six decreasing values. For six of the 20 participants, no AE was present and the maximum $\mathrm{AE}$ value was set to 0 , which, if anything, should work against finding a reliable maximum AE. Nevertheless, a two-tailed $t$ test on these values revealed that they significantly differed from $0, t(19)=3.75, P<0.01$. For the 16 participants that showed an $\mathrm{AE}$, the mean size of the effect was $0.90 \mathrm{~mm}$.

With shorter stimulus motion/target movement durations (i.e., higher speed stress) and the absence of advance information about the direction of stimulus motion prior to movement onset, an AE was observed in the initial portion of the movement trajectories. This result was to be expected based on the facilitation effects that have generally been observed in continuous S-R compatibility tasks (e.g., Chua \& Weeks, 1997; Keller \& Burnham, 2005; Michaels \& Stins, 1997) and the kinematics of aimed hand movements in the presence of unrelated visual motion (e.g., Whitney et al., 2003). As in "Experiment 1", a CE was observed in later portions of the trajectories.

\section{General discussion}

The aim of this study was to further our understanding of specific interactions between perception and functionally unrelated ongoing action. Participants were asked to move one of their hands (without visual feedback) in a certain direction while concurrently observing a stimulus motion of a (dis)similar direction. The results of "Experiment 1" revealed a $\mathrm{CE}$, in that having watched, for example, an upward motion led to a lower movement endpoint than having watched a downward motion. This finding generalizes those of Schubö et al. (2001) to (a) the overlapping motion/ movement dimension of direction and to (b) the use of nonbiological stimulus motions. The latter finding is of interest because previous studies often relied on the presentation of biological stimulus motions (see also Kilner et al., 2003), which are known to be processed differently than motions with biological velocity profiles (e.g., Viviani, 2002). What is more, interference effects obtained in similar paradigms have been attributed to the involvement of the motor system in the visual processing of biological motion (e.g., Kilner et al., 2003). The present results suggest that this is not necessarily the case.

With higher speed stress and no advance information pertaining to the direction of stimulus motion, the CE of "Experiment 1" was replicated in "Experiment 2". This time, however, two additional effects were observed. First, movement times were slower under incompatible motion/ movement direction conditions, which resembles the response slowing that is generally observed under spatially incompatible $\mathrm{S}-\mathrm{R}$ relationships in reaction-time tasks (e.g., Simon, 1990). The second novel effect to arise in "Experiment 2" was an AE in the initial portions of the movement trajectories. This result was to be expected based on the facilitation effects that have generally been observed in continuous S-R compatibility tasks (e.g., Chua \& Weeks, 1997; Keller \& Burnham, 2005; Michaels \& Stins, 1997). The absence of these two effects in "Experiment 1" could be related to the fact that that movement onset was preceded by stimulus motion onset and/or to the use of relatively lenient movement speed constraints in that experiment. Both of these factors should have reduced participants' ability to engage in compensatory movements aimed at countering any anticipated or perceived interference effects.

More generally, the present results revealed a bi-phasic pattern of interference of perception on ongoing action: initial assimilation followed by contrast. There are two main lines of theoretical thinking that could account for these effects. The first holds that the $\mathrm{AE}$ and $\mathrm{CE}$ are reflections of sensorimotor adjustments that are made on the basis of perceived discrepancies between motor-specific sensory predictions and incoming sensory feedback. The second line of thinking attributes these effects to the activation and inhibition of the representations underlying perception and/or action.

\section{Sensorimotor adjustments}

The AE was observed after an interval $(\sim 110 \mathrm{~ms})$ that is consistent with other estimates of fast visuomotor delays (e.g., Brenner \& Smeets, 1997; Desmurget \& Grafton, 2000; Whitney et al., 2003). Among the various types of tasks employed to estimate such delays, the study of Whitney et al. (2003) is particularly relevant here. They reported that when people try to reach as quickly as possible to a briefly flashed stationary target in the presence of an unrelated moving grating, they initially move their hand in the direction of motion. They took this AE as evidence of an adaptive visuomotor mechanism that rapidly alters hand trajectories under conditions of perceived ego-motion. Since retinal motion is most often associated with eye and head movements, they argued that the background (i.e., grating) motion could have induced an illusory sense of ego-motion. Thus, for example, upward motion would (normally) be associated with downward eye/head movements (and a perceived downward sense of ego-motion), which would result in an upward adjustment of the hand trajectory. Although their task was in many ways different than 
that employed in the current study, their interpretation could be applied here as well. Nevertheless, it is unclear whether the stimulus motions in the current study were sufficient to produce the type of ego-motion that may have been induced by the moving grating in Whitney et al.

The CEs were observed in later portions of the trajectories, $\sim 260 \mathrm{~ms}$ after movement/motion onset at the earliest. This delay is consistent with the time it takes to engage in voluntary visuomotor adjustments (e.g., Desmurget \& Grafton, 2000; Paillard, 1996). One interpretation of the CE is that, despite having been told otherwise, participants may have considered the trajectory they observed as a consequence of the movement they executed. If this was the case, they may have attempted to correct for the perceived discrepancy between the trajectory they wanted to produce and the trajectory they thought they were actually producing. The fact that participants can correct online for visual perturbations of hand trajectories is well documented (e.g., Fourneret \& Jeannerod, 1998).

There are, however, a number of reasons why this interpretation seems unlikely. For one, we employed three spatially distinct straight-line stimulus motion trajectories that had velocity profiles uncharacteristic of biological movements. Furthermore, the motions started on average $\sim 230 \mathrm{~ms}$ prior to the movements in "Experiment 1", thereby preceding any overt motor activity. Lastly, to rule out this account of the CE, Schubö et al. (2001) performed another experiment in which the stimulus and movement for a given trial were staggered in time by up to $500 \mathrm{~ms}$, such that it became highly unlikely that the observed motion represented the sensory consequence of the movement that was being performed. Under such conditions, a CE was nonetheless obtained. Although, we cannot fully rule out this account, the similarity between the CEs observed by Schubö et al. (2001) and ourselves further suggests that this is not the primary mechanism at work.

An alternative type of adjustment that could have produced the CE is more "strategic" in nature. Specifically, participants may have become aware of their tendency to show an AE (independently of its origin) and voluntarily engaged in compensatory movements that ultimately lead to a CE. Given the plausibility of this explanation, an object of future research should be to systematically assess participants' awareness of any signs of interference and determine whether it is systematically related to the presence of the CE.

\section{Activation and inhibition}

An alternative account of the AE and CE is that they reflect the type of processes that are generally thought to lead to specific interference effects in S-R compatibility tasks: The automatic activation and subsequent inhibition of actionrelated representational features (e.g., see Kornblum, Stevens, Whipple, \& Requin, 1999; Prinz \& Hommel, 2002). For example, using static visual displays, Tipper, Howard, and Jackson (1997) have shown that hand trajectories to a target object veer away from the location of a concurrently presented distractor object. Adopting the notion that movement directions are represented in a distributed fashion, such that neighboring directions are also partially active (cf. Georgopoulos, 1990), Tipper et al. (1998) proposed that perception of the target and distractor objects automatically activates representations underlying movements in their direction. In order to select the appropriate response, the representation corresponding to the distractor was assumed to be inhibited, thereby inhibiting any shared directional features that were activated by the perception of the target object. As a consequence, movement trajectories tend to veer away from the distractor location (see also Welsh \& Elliott, 2004). Note that this type explanation does not rely on an adjustment based on a perceived discrepancy, as discussed in the previous section, but rather on a shift of the representation underlying the produced movement direction itself.

Schubö et al. (2001) relied on similar assumptions to account for their CE. They proposed that the distributed representations that underlie distinct perceptual and motor activities, such as producing a movement while concurrently encoding an independent stimulus motion, must be "kept separate" or "protected" from one another so that the two activities can be carried out without interfering. More specifically, they assumed that perception and action share a common representational system (Prinz, 1990, 1997) in which the simultaneous perception and production of feature-overlapping events becomes potentially problematic (for more detailed discussions of this issue, see Hommel, Müsseler, Aschersleben, \& Prinz, 2001). To deal with such problems, they proposed that a selective code-modification mechanism comes into play to increase the distinctiveness of the representations underlying the two activities. The mechanism in question was posited to involve a form of partial inhibition (Tipper et al., 1997) of the features shared by perception and action, which causes the representations underlying them to "shift away" from each other within the common representational space. Hence, what participants perceived had a repulsive effect on what they produced. This mechanism could have come into play after whatever mechanism lead to the initial AE.

The $\mathrm{AE}$ is also related to an effect that has been reported in the selective-reaching task considered above (e.g., Welsh, Elliott, \& Weeks, 1999). Hand trajectories have been found to veer towards the distractor location under certain conditions. This effect has been attributed to an initial summation of the activations of the directional features 
shared by the target and distractor representations, which causes the movement to the target to be biased in the direction of the distractor location. Welsh and Elliott (2004) have proposed that this effect should only manifest itself when there is not enough time to inhibit the distractor representation, which also explains why it has not always been observed (for a detailed discussion of this issue, see Welsh \& Elliott, 2004).

The AE found in the present study could be explained in a similar way. That is, the initial activation/summation process could shift the representation corresponding to the produced movement in the direction of the perceived motion direction. Given that the stimulus motion started prior to movement onset in "Experiment 1", the absence of an $\mathrm{AE}$ in that experiment suggests that there was enough time for the subsequent inhibition process to engage prior to the initial phase of movement. Thus, only a CE was observed. To the extent that these accounts of the AE and CE hold, the bi-phasic pattern of interference obtained in "Experiment 2 " should reflect the (average) time course of the activation/ summation and subsequent inhibition of the representations underlying perception and action.

As it stands, however, both sensorimotor-adjustment and activation-inhibition accounts seem to provide viable explanations, in isolation or combination, of the basic effects obtained in this study. Thus, additional work will be needed to better establish the origins of the bi-phasic pattern of specific interference. For example, one could compare the nature and time course of the interference effects obtained with moving and static stimuli, such as the appearance of a line or arrow. The latter type of stimuli should no longer induce a sense of ego-motion and would therefore allow one to test whether this sense is actually what underlies the $\mathrm{AE}$ reported here.

Acknowledgments We would like to thank three anonymous reviewers for constructive comments, Eric Grosjean for valuable advice on the data analysis, Wilfried Kunde for providing code to collect data from the graphics tablet, Fiorello Banci for technical support, and Silvia Bauer and Cornelia Maier for assistance with running the participants.

\section{References}

Beggs, W. D. A., \& Howarth, C. I. (1972). The movement of the hand towards a target. Quarterly Journal of Experimental Psychology, $24,448-453$

Brass, M., Bekkering, H., Wohlschläger, A., \& Prinz, W. (2000). Compatibility between observed and executed finger movements: Comparing symbolic, spatial, and imitative cues. Brain and Cognition, 44, 124-143.

Brenner, E., \& Smeets, J. B. J. (1997). Fast responses of the human hand to changes in target position. Journal of Motor Behavior, 29, 297-310.

Chua, R., \& Weeks, D. J. (1997). Dynamical explorations of compatibility in perception-action coupling. In B. Hommel, \& W. Prinz
(Eds.), Theoretical issues in stimulus-response compatibility (pp. 373-398). Amsterdam: North-Holland.

De Jong, R., Liang, C.-C., \& Lauber, E. (1994). Conditional and unconditional automaticity: A dual-process model of effects of spatial stimulus-response compatibility. Journal of Experimental Psychology: Human Perception and Performance, 20, 731750 .

Desmurget, M., \& Grafton, S. (2000). Forward modeling allows feedback control for fast reaching movements. Trends in Cognitive Sciences, 4, 423-431.

Fourneret, P., \& Jeannerod, M. (1998). Limited conscious monitoring of motor performance in normal subjects. Neuropsychologia, 36, $1133-1140$.

Georgopoulos, A. P. (1990). Neurophysiology of reaching. In M. Jeannerod (Ed.), Attention and performance XIII (pp. 227-263). Hillsdale, NJ: Lawrence Erlbaum Associates.

Grosjean, M., \& Mordkoff, J. T. (2001). Temporal stimulus-response compatibility. Journal of Experimental Psychology: Human Perception and Performance, 27, 870-878.

Hamilton, A., Wolpert, D., \& Frith, U. (2004). Your own action influences how you perceive another person's action. Current Biology, 14, 493-498.

Hommel, B., Müsseler, J., Aschersleben, G., \& Prinz, W. (2001). The theory of event coding (TEC): A framework for perception and action planning. Behavioral and Brain Sciences, 24, 849-937.

Hommel, B., \& Prinz, W. (Eds.). (1997). Theoretical issues in stimulus-response compatibility. Amsterdam: North-Holland.

Jacobs, A., \& Shiffrar, M. (2005). Walking perception by walking observers. Journal of Experimental Psychology: Human Perception and Performance, 31, 157-169.

Keller, P. E., \& Burnham, D. K. (2005). Musical meter in attention to multipart rhythm. Music Perception, 22, 629-661.

Kilner, J. M., Paulignan, Y., \& Blakemore, S.-J. (2003). An interference effect of observed biological movement on action. Current Biology, 13, 522-525.

Kornblum, S., Stevens, G. T., Whipple, A., \& Requin, J. (1999). The effects of irrelevant stimuli: 1 . The time course of stimulus-stimulus and stimulus-response consistency effects with Stroop-like stimuli, Simon-like tasks, and their factorial combinations Journal of Experimental Psychology: Human Perception and Performance, 25, 688-714.

Michaels, C. F., \& Stins, J. F. (1997). An ecological approach to stimulus-response compatibility. In B. Hommel, \& W. Prinz (Eds.), Theoretical issues in stimulus-response compatibility (pp. 333360). Amsterdam: Elsevier.

Morasso, P. (1981). Spatial control of arm movements. Experimental Brain Research, 42, 223-227.

Müsseler, J. (1999). How independent from action control is perception? An event-coding account for more equally-ranked crosstalks. In: G. Aschersleben, T. Bachmann, \& J. Müsseler (Eds.), Cognitive contributions to the perception of spatial and temporal events (pp. 121-147). Amsterdam: Elsevier.

Paillard, J. (1996). Fast and slow feedback loops for the visual correction of spatial errors in a pointing task: A reappraisal. Canadian Journal of Physiology and Pharmacology, 74, 401-417.

Prinz, W. (1990). A common coding approach to perception and action. In: O. Neumann, \& W. Prinz (Eds.), Relationships between perception and action: Current approaches (pp. 167-201). Berlin: Springer.

Prinz, W. (1997). Perception and action planning. European Journal of Cognitive Psychology, 9, 129-154.

Prinz, W., \& Hommel, B. (Eds.). (2002). Common mechanisms in perception and action. Attention and performance XIX. New York, NY: Oxford University Press.

Proctor, R. W., \& Reeve, T. G. (Eds.). (1990). Stimulus-response compatibility: An integrated perspective. Amsterdam: North-Holland. 
Rubichi, S., Nicoletti, R., Umiltà, C., \& Zorzi, M. (2000). Response strategies and the Simon effect. Psychological Research, 63, 129136.

Saunders, J. A., \& Knill, D. C. (2003). Humans use continuous visual feedback from the hand to control fast reaching movements. Experimental Brain Research, 152, 341-352.

Schmidt, R. A., \& Lee, T. D. (1999). Motor control and learning: A behavioral emphasis (3rd ed.). Champaign, IL: Human Kinetics.

Schubö, A., Aschersleben, G., \& Prinz, W. (2001). Interactions between perception and action in a reaction task with overlapping S-R assignments. Psychological Research, 65, 145-157.

Schubö, A., Prinz, W., \& Aschersleben, G. (2004). Perceiving while acting: Action affects perception. Psychological Research, 68, 208-215.

Simon, J. R. (1990). The effects of an irrelevant directional cue on human information processing. In R. W. Proctor, \&T. G. Reeve (Eds.), Stimulus-response compatibility: An integrated perspective (pp. 31-86). Amsterdam: North-Holland.

Spivey, M. J., Grosjean, M., \& Knoblich, G. (2005). Continuous attraction towards phonological competitors. Proceedings of the $\mathrm{Na}$ tional Academy of Sciences, 102, 10393-10398.

Tipper, S. P., Howard, L. A., \& Houghton, G. (1998). Action-based mechanisms of attention. Philosophical Transactions of the Royal Society, 353, 1385-1393.

Tipper, S. P., Howard, L. A., \& Jackson, S. R. (1997). Selective reaching to grasp: Evidence for distractor interference effects. Visual Cognition, 4, 1-38.
Viviani, P. (2002). Motor competence in the perception of dynamic events: A tutorial. In: W. Prinz \& B. Hommel (Eds.), Common mechanisms in perception and action. Attention and performance (Vol. XIX, pp. 406-442). New York, NY: Oxford University Press.

Welsh, T. N., \& Elliott, D. (2004). Movement trajectories in the presence of a distracting stimulus: Evidence for a response activation model of selective reaching. The Quarterly Journal of Experimental Psychology, 57A, 1031-1057.

Welsh, T. N., Elliott, D., \& Weeks, D. J. (1999). Hand deviations toward distractors: Evidence for response competition. Experimental Brain Research, 127, 207-212.

Whitney, D., Westwood, D. A., \& Goodale, M. A. (2003). The influence of visual motion on fast reaching movements to a stationary object. Nature, 423, 869-873.

Wiegand, K., \& Wascher, E. (2005). Dynamic aspects of stimulus-response correspondence: Evidence for two mechanisms involved in the Simon effect. Journal of Experimental Psychology: Human Perception and Performance, 31, 453-464.

Wohlschläger, A. (2000). Visual motion priming by invisible actions. Vision Research, 40, 925-930.

Zwickel, J., Grosjean, M., \& Prinz, W. (2007). Seeing while moving: Measuring the online influence of action on perception. Quarterly Journal of Experimental Psychology, 60, 1063-1071. 